\title{
Micotoxinas na dieta de bovinos de corte: revisão
}

\author{
Vedovatto, M.G.; Bento, A.L.; Kiefer, C.; Souza, K.M.R. e Franco, G.L.@
}

Universidade Federal de Mato Grosso do Sul. UFMS. Campus Universitário de Campo Grande. MS. Brasil.

\section{PALAVRAS CHAVE ADICIONAIS}

\section{Aflatoxina.}

Ocratoxin.

Ruminantes.

Tricotecenos.

Zearalenona.

\section{RESUMO}

Esta revisão foi realizada com o objetivo de fazer um breve histórico, caracterizar, descrever as alterações metabólicas, sinais clínicos, métodos de prevenção, controle e detoxificação das principais micotoxinas consumidas por bovinos de corte. Após a descoberta da aflatoxina e dos problemas que esta pode causar para os animais e também em humanos, principalmente câncer, as pesquisas cientificas aumentaram ano após ano, e hoje são descritos mais de 18000 metabólitos secundários produzidos por fungos, porém os mais estudados são: aflatoxinas, ocratoxina $A$, tricotecenos, zearalenona, patulina, fumonisina e alcaloides de Ergot. Os ruminantes de uma forma geral necessitam de maiores concentrações de micotoxinas na dieta quando comparados aos monogástricos para apresentarem sintomas clínicos de intoxicação. Isso acontece pelo fato de que algumas de micotoxinas podem ser parcialmente ou totalmente degradadas pelos microrganismos ruminais. Porém, a fermentação ruminal não resulta necessariamente em inativação da toxina, e a extensão da metabolização depende além do tipo de micotoxina consumida, da espécie animal, idade, sexo, raça, tipo de dieta e consequentemente dos tipos de microrganismos que habitam o rúmen. Além disso, algumas micotoxinas possuem ação antimicrobiana, e podem alterar negativamente o metabolismo ruminal. A presença de micotoxinas na dieta pode afetar além da saúde o desempenho produtivo e reprodutivo o que pode levar a altas perdas econômicas. Assim os sistemas de produção de alimentos para bovinos devem adotar práticas agrícolas que minimizem a produção desses metabólitos.

\section{Mycotoxins in the beef cattle diet. review}

\section{SUMMARY}

\section{ADDITIONAL KEYWORDS}

Aflatoxins.

Ochratoxin.

Ruminants.

Trichothecenes.

Zearalenone.

\section{INFORMATION}

Cronología del artículo.

Recibido/Received: 15.09.2018

Aceptado/Accepted: 05.03.2020

On-line: 15.04.2020

Correspondencia a los autores/Contact e-mail:

gumercindo.franco@ufms.br

\section{INTRODUÇÃO}

Micotoxinas são metabólitos secundários produzidos por fungos filamentosos que causam respostas tóxicas quando ingeridas por humanos ou animais. Espécies de Fusarium, Aspergillus e Penicillium são os mais abundantes fungos que contaminam os alimentos e produzem micotoxinas antes, durante e após a colhei- ta e também devido o armazenamento inadequado dos alimentos (Binder, 2007, p. 149).

Bovinos que consomem dietas contaminadas com micotoxinas podem apresentar intoxicação aguda ou crônica, com efeitos que podem ser teratogênicos, carcinogênicos, estrogênicos ou imunossupressores. Consequências diretas do consumo de dietas contaminadas 
incluem: redução no consumo de matéria seca, recusa da dieta, baixa conversão alimentar, redução no ganho de peso, aumento de incidência de doenças (devido à imunossupressão) e redução do desempenho reprodutivo, o que pode levar a altas perdas econômicas (Binder et al., 2007, p. 150). As micotoxinas de maior importância para bovinos de corte são: aflatoxinas, ocratoxina $\mathrm{A}$, tricotecenos, zearalenona, patulina, fumonisina e alcaloides de Ergot (Hussein e Brasel, 2001, p.101). Estas apresentam mecanismos de ação, alterações metabólicas e sinais clínicos específicos para cada uma.

Os ruminantes são menos susceptíveis que as outras espécies quanto às intoxicações por micotoxinas (Reisinger et al., 2019, pp. 1). Está hipótese baseia-se na constatação de que os microrganismos ruminais podem converter algumas micotoxinas em metabólitos menos tóxicos ou até mesmo inativá-los (Reisinger et al., 2019, pp. 1). Porém, essa regra não se aplica a todas as micotoxinas, pois algumas são pouco biodegradadas e por possuírem efeito antimicrobiano alteram negativamente a fermentação ruminal (Fink-Gremmels, 2008b, p. 84; Reisinger et al., 2019, pp. 1).

Assim esta revisão foi realizada com o objetivo de fazer um breve histórico, caracterizar, descrever as alterações metabólicas, sinais clínicos, métodos de prevenção, controle e detoxificação das principais micotoxinas consumidas por bovinos de corte.

\section{BREVE HISTÓRICO E SITUAÇÃO ATUAL DAS PESQUI- SAS SOBRE MICOTOXINAS}

No início da década de 60 Stevens et al. (1960, p. 627) descreveram o aparecimento de uma nova doença em aves. Estas apresentavam perda de apetite, redução na mobilidade, fraqueza nas asas e pernas e morriam em aproximadamente uma semana após o início desses sintomas. Nas necropsias eram observadas lesões necróticas no fígado e congestionamento dos rins. Os autores não conseguiram isolar o agente infeccioso e suspeitaram que essa doença pudesse ser de origem nutricional, pois com a mudança da ração cessavam os sintomas.

Em 1960 na Inglaterra foi observado que o fornecimento de torta de amendoim (Arachis hypogaea) proveniente do Brasil causou mortes de aves (Blount, 1960, p. 786). Este autor realizou diversas análises, com o objetivo de identificar a presença de inseticidas, raticidas, chumbo, entre outras e observou que quando algumas destas substâncias eram encontradas elas não estavam em níveis tóxicos. Assim Blount (1961, p. 52) por não identificar o agente causador da doença, denominou esta de "Turkey X disease" e foi responsabilizada pela morte de mais de 100000 aves entre maio a agosto de 1960.

Em 1961 foi publicado na revista "Nature" um trabalho (Sargeant et al., 1961, p. 1096) relatando a ocorrência de mortes de perus, marrecos, bovinos, ovinos e suínos na Inglaterra e que consumiram torta de amendoim proveniente do Brasil. Os mesmos autores conseguiram purificar extratos tóxicos desta torta de amendoim e obtiveram uma solução cristalina. Estes forneceram $20 \mu \mathrm{g}$ desta para marrequinhos e observaram que morriam em média 24 horas após o fornecimento e também foram encontradas lesões no fígado. Suspeitando que a intoxicação pudesse ser causada por fungos, estes autores produziram culturas puras de fungos e posteriormente multiplicaram estes em torta de amendoim previamente esterilizada. $\mathrm{O}$ fornecimento desta para marrequinhos resultou em mortes com as mesmas lesões no fígado. O fungo produzido foi identificado como Aspergillus flavus Link ex Fries e a toxina denominada Aflatoxina.

Após a descoberta da aflatoxina e dos problemas que esta pode causar para animais de interesse zootécnico e também em humanos, principalmente câncer, as pesquisas científicas aumentaram ano após ano. No banco de dados Scopus somente no ano de 2019 foram encontrados 920, 21 e 23 artigos para as buscas com as palavras-chave "Mycotoxin", "Mycotoxin and Cattle" e "Mycotoxin and Silage", respectivamente. Se somarmos desde 1960 até 2019 são encontrados 17829, 578 e 195 para as buscas com as mesmas palavras-chave (Figura 1). Isto demonstra a dimensão da importância e do interesse da comunidade científica pelo assunto.

Além de micotoxinas produzidas por fungos do gênero Aspergillus, até o momento já foram identificados compostos secundários produzidos por Fusarium, Penicillium, Alternaria, Chaetomium, Cladosporium, Claviceps, Diplodia, Myrothecium, Monascus, Phoma, Phomopsis, Pithomyces, Trichoderma e Stachybotrys. Atualmente foram descritos cerca de 18000 metabólitos secundários de fungos, porém os mais estudados são: aflatoxinas, ocratoxina A, tricotecenos, zearalenona, patulina, fumonisina e alcaloides de Ergot (Gallo et al., 2015, p. 3058).

\section{CARACTERÍSTICAS DAS PRINCIPAIS MICOTOXINAS}

As micotoxinas são metabólitos secundários produzidos pelo micélio de fungos filamentosos e que não tem nenhuma função bioquímica no crescimento e desenvolvimento dos fungos (Moss, 1991, p. 37). A razão exata porque os fungos produzem micotoxinas não é completamente entendida. Algumas teorias apontam para uma possível competição do fungo contra os mecanismos de defesa das plantas ou auxiliando os fungos a competir em seu nicho ecológico, por exemplo, algumas micotoxinas são antibacterianas e esses metabólitos podem reduzir o número de microrganismos competindo pelo mesmo substrato (Richard, 2012, p. 2).

As micotoxinas podem ser classificadas em função da espécie de fungo, estrutura e modo de ação. Uma mesma espécie de fungo pode ser produtora de diferentes micotoxinas, dependendo da condição ambiental, assim como uma mesma micotoxina pode ser sintetizada por diferentes espécies de fungos. Existe uma grande quantidade de fungos que produzem micotoxinas, porém os gêneros de maior importância zootécnica são Aspergillus, Penicillium e Fusarium. As micotoxinas podem apresentar uma grande variedade de conformações estruturais (Hussein e Brasel, 2001, p. 104) e as de maior importância para bovinos são: 


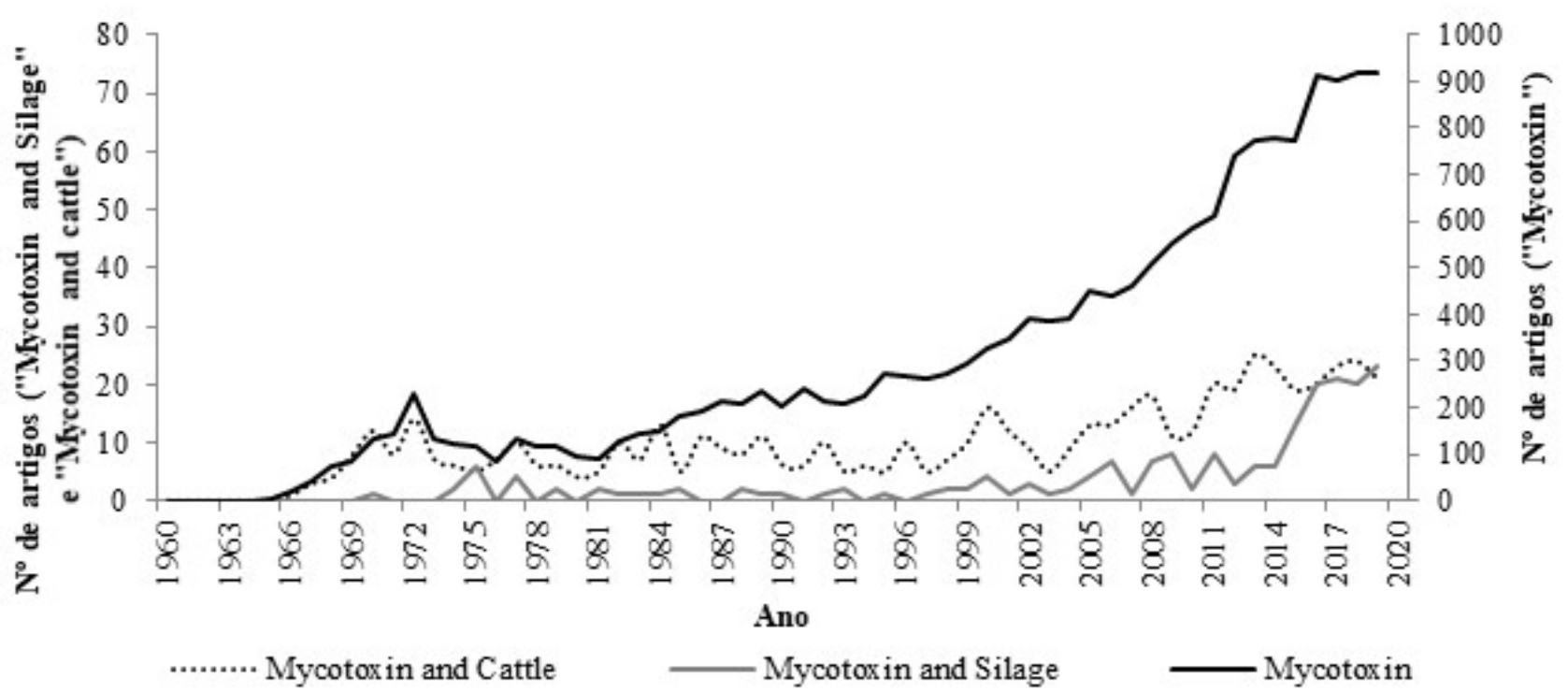

Figura 1. Número de artigos (incluindo revisões de literatura) do banco de dados Scopus obtidos através da busca pelas palavras-chave "Mycotoxin", "Mycotoxin and Cattle" $\mathrm{e}$ "Mycotoxin and silage" presentes no título, resumo ou palavras-chave dos trabalhos publicados entre os anos de 1960 e 2019 (Number of articles (including literature reviews) from the Scopus database obtained by searching for the keywords "Mycotoxin", "Mycotoxin and Cattle" and "Mycotoxin and silage" in the title, abstract or keywords of the papers published between 1960 and 2019).

\section{BIOTRANSFORMAÇÕES E ALTERAÇÕES NO METABO- LISMO RUMINAL}

Os ruminantes de uma forma geral necessitam de maiores concentrações de micotoxinas nas dietas quando comparados aos monogástricos para apresentar sintomas clínicos de intoxicação. Isso acontece pelo fato de que algumas micotoxinas podem ser parcialmente ou totalmente degradadas pelos microrganismos ruminais. Porém a fermentação ruminal não resulta necessariamente em inativação da toxina, e a extensão da metabolização depende do tipo de micotoxina consumida, da espécie animal, idade, sexo, raça, dieta e consequentemente dos tipos de microrganismos que habitam o rúmen (Upadhaya et al., 2009, p. 29). Além disso, algumas micotoxinas possuem ação antimicrobiana, e podem alterar negativamente o metabolismo ruminal (Fink-Gremmels, $2008^{\mathrm{a}}$, p. 174).

\section{AfLATOXINAS}

As aflatoxinas geralmente são pouco degradas no rúmen. Em um experimento in vitro somente $10-14 \%$ da aflatoxina foi degradada no líquido ruminal de novilhos alimentados com uma relação volumoso:concentrado de 60:40 (Upadhaya et al., 2009, p. 30). Além disso a aflatoxina que é degradada (AFB1) é convertida para aflatoxicol que é um composto altamente toxico para as bactérias afetando o crescimento e atividade metabólica dos microrganismos do rúmen. Muitas bactérias são completamente inibidas por concentrações de AFB1 próximas a $10 \mu \mathrm{g} / \mathrm{mL}$ (Auerbach et al., 1998, p. 565).

Em um trabalho in vitro conduzido por Jiang et al. (2012, p. 85) foram testadas no líquido ruminal de bovinos que consumiam uma dieta a base de feno de aveia e farelo de milho (75:25) diferentes doses de AFB1 $(0,320,640$ e $960 \mathrm{ng} / \mathrm{mL})$ sobre a fermentação ruminal. Comparando a maior dose $(960 \mathrm{ng} / \mathrm{mL})$ ao controle $(0 \mathrm{ng} / \mathrm{mL})$ não foram observadas alterações na digestibilidade in vitro da matéria seca, porém houve redução na taxa de produção de gás $(0,16$ e 0,22 , respectivamente), nitrogênio amoniacal (17,2 e 19,5 mM, respectivamente) e na produção total de AGV $(87,1$ e 99,8 mM, respectivamente). Além destes efeitos, em um estudo mais antigo executado por Cook et al. (1986, p. 1816) foram observadas redução da motilidade ruminal de vacas que receberam uma administração oral de 0,2 a 0,8 ppm de AFB1. Esses resultados demonstram que apesar da aflatoxina ser pouco degradada no rúmen, dependendo da quantidade presente na dieta o metabolismo ruminal pode ser altamente alterado.

\section{OCRATOXINA A}

A ocratoxina A pode ser altamente degradada no rúmen e ocorre redução do seu efeito tóxico. Esta é um composto complexo formado por uma ocratoxina $\alpha(\mathrm{OT} \alpha)$ ligada através de um grupo 7-carboxílico a L$\beta$-fenilalanina por uma ligação amida. A hidrólise desta última pelos microrganismos ruminais leva a liberação da OT $\alpha$ e da fenilalanina, que desta forma são considerados menos ou até mesmo não tóxicos

\section{BIOTRANSFORMAÇÕES E ALTERAÇÕES NO METABO- LISMO RUMINAL}

Os ruminantes de uma forma geral necessitam de maiores concentrações de micotoxinas nas dietas quando comparados aos monogástricos para apresentar sintomas clínicos de intoxicação. Isso acontece pelo fato de que algumas micotoxinas podem ser parcialmente ou totalmente degradadas pelos microrganismos ruminais. Porém a fermentação ruminal não resulta necessariamente em inativação da toxina, e a extensão da metabolização depende do tipo de micotoxina consumida, da espécie animal, idade, sexo, raça, dieta 
Tabela I. Características das principais micotoxinas que causam intoxicação em bovinos de corte (Characteristics of the main mycotoxins that cause intoxication in beef cattle)

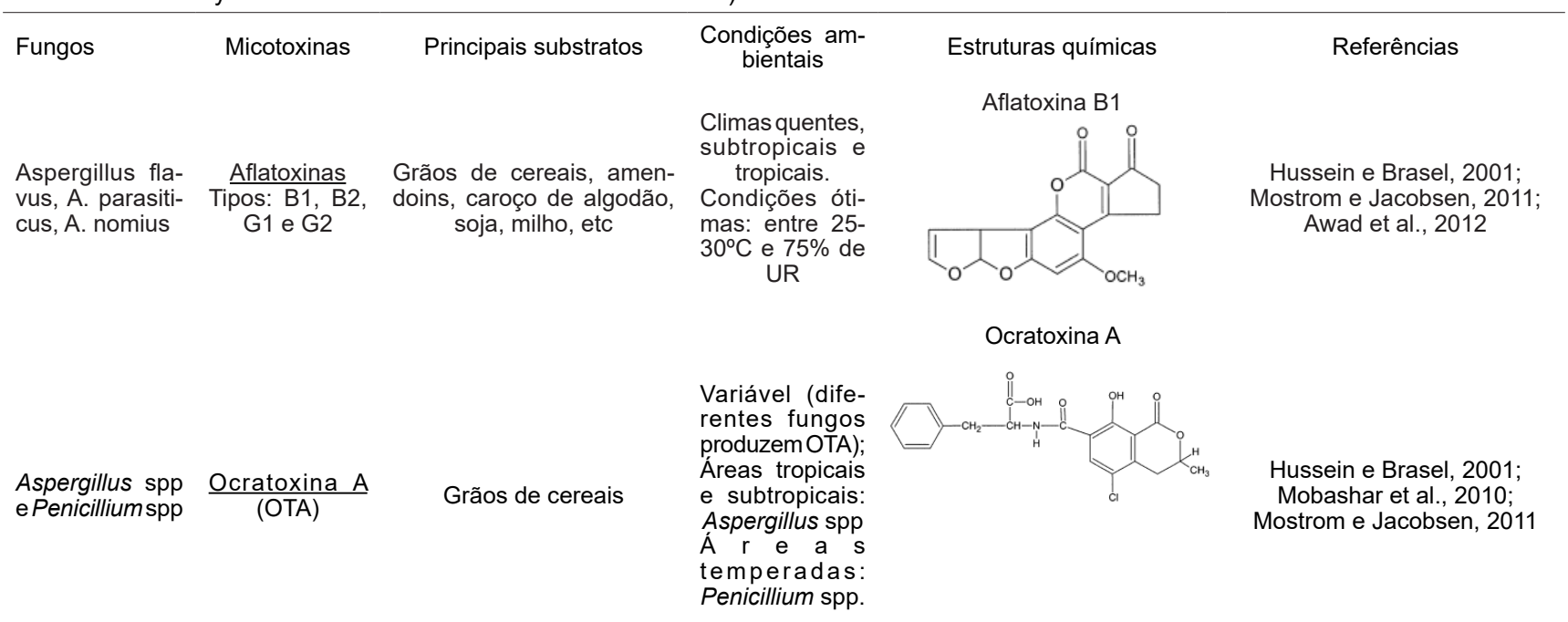

Deoxivalenol

Variável: tipo tricoteceno pode variar de acordo a temperatura: Ex:F. graminearum em épocas frias produz mais deoxivalenol e em épocas quentes e chuvosas nivalenol

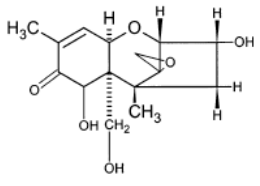

Hussein e Brasel, 2001; Mostrom e Jacobsen, 2011;

Fruhmann et al., 2014
Fusarium Spp:F. graminearum (Gibberella zeae), F. culmorum, F. cerealis, $F$. equiseti e $F$ semitectum

Penicillium spp; Aspergillus spp Byssochlamus spp

Zearalenona

Grãos de cereais, como trigo, cevada, arroz, milho e também em feno

Resíduos de destilarias, subprodutos de frutas (principalmente macã)e também

$\underline{\text { Patulina }}$

Fumonisina

Fusarium (F. Tipos: B 1 $\begin{array}{ll}\text { proliferatum e } & \text { (maior impor- } \\ F \text {. verticillioides) } & \text { tância zootéc- }\end{array}$ nica) e B2

Alcaloides de

$$
\text { Ergot }
$$

Tipos: alca loides de erClaviceps spp e gopeptina (erspp govalina, ergovalina, etc) e alcaloides de govina, ácido lisérgico, etc)

Alta umidade e temperatura em silagem
Forragens vivas (principalmente em Festuca (Lolium arundinaceum), mas também em grãos de cereais
Principalmente em climas temperados

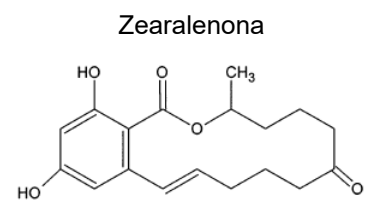

Patulina

Variáveis:

Alterações climáticas (estresse). Ex: período de seca durante a fase de desen-

Grãos de cereais, principalvolvimento do milho seguido por chuva ou frio durante a polinização estimulação a produção de fumonisinas

Ergotamina

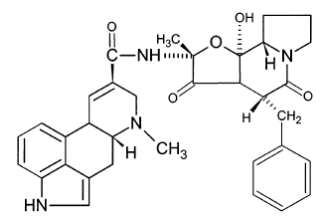

Placinta et al., 1999; Hussein e Brasel, 2001; Mostrom e Jacobsen, 2011; Krížová et al., 2012

Moake et al., 2005; Fink-Gremmels, 2008 Mostrom e Jacobsen, 2011

Hussein e Brasel, 2001; Mostrom e Jacobsen, 2011

Hussein e Brasel, 2001: Mostrom e Jacobsen, 2011; Strickland et al., 2011 
e consequentemente dos tipos de microrganismos que habitam o rúmen (Upadhaya et al., 2009, p. 29). Além disso, algumas micotoxinas possuem ação antimicrobiana, e podem alterar negativamente o metabolismo ruminal (Fink-Gremmels, 2008 ${ }^{\mathrm{a}}$, p. 174).

\section{AfLATOXINAS}

As aflatoxinas geralmente são pouco degradas no rúmen. Em um experimento in vitro somente $10-14 \%$ da aflatoxina foi degradada no líquido ruminal de novilhos alimentados com uma relação volumoso:concentrado de 60:40 (Upadhaya et al., 2009, p. 30). Além disso a aflatoxina que é degradada (AFB1) é convertida para aflatoxicol que é um composto altamente toxico para as bactérias afetando o crescimento e atividade metabólica dos microrganismos do rúmen. Muitas bactérias são completamente inibidas por concentrações de AFB1 próximas a $10 \mu \mathrm{g} / \mathrm{mL}$ (Auerbach et al., 1998, p. 565).

Em um trabalho in vitro conduzido por Jiang et al. (2012, p. 85) foram testadas no líquido ruminal de bovinos que consumiam uma dieta a base de feno de aveia e farelo de milho (75:25) diferentes doses de AFB1 (0, 320, 640 e $960 \mathrm{ng} / \mathrm{mL})$ sobre a fermentação ruminal. Comparando a maior dose $(960 \mathrm{ng} / \mathrm{mL}$ ) ao controle $(0 \mathrm{ng} / \mathrm{mL})$ não foram observadas alterações na digestibilidade in vitro da matéria seca, porém houve redução na taxa de produção de gás $(0,16$ e 0,22, respectivamente), nitrogênio amoniacal (17,2 e 19,5 mM, respectivamente) e na produção total de AGV $(87,1$ e 99,8 mM, respectivamente). Além destes efeitos, em um estudo mais antigo executado por Cook et al. (1986, p. 1816) foram observadas redução da motilidade ruminal de vacas que receberam uma administração oral de 0,2 a 0,8 ppm de AFB1. Esses resultados demonstram que apesar da aflatoxina ser pouco degradada no rúmen, dependendo da quantidade presente na dieta o metabolismo ruminal pode ser altamente alterado.

\section{OCRATOXINA A}

A ocratoxina A pode ser altamente degradada no rúmen e ocorre redução do seu efeito tóxico. Esta é um composto complexo formado por uma ocratoxina $\alpha(\mathrm{OT} \alpha)$ ligada através de um grupo 7-carboxílico a L- $\beta$-fenilalanina por uma ligação amida. A hidrólise desta última pelos microrganismos ruminais leva a liberação da OT $\alpha$ e da fenilalanina, que desta forma são considerados menos ou até mesmo não tóxicos (Sreemannarayana et al., 1988, p. 1704). As enzimas proteolíticas, e principalmente as carboxipeptidases são as principais enzimas envolvidas na degradação da ocratoxina A (Mobashar et al., 2010, p. 815).

Estudos in vitro mostraram que a ocratoxina A é degradada principalmente pelos protozoários do rúmen, e que em bovinos saudáveis até $12 \mathrm{mg}$ desta por $\mathrm{kg}$ de ração podem ser inativados (Hult et al., 1976, p. 443). A velocidade de degradação depende do tipo de animal, e principalmente do tipo da dieta (a degradação aumenta com o aumento da quantidade de concentrado). Normalmente a ocratoxina A apresenta meia vida no rúmen de menos de 2,76 horas (Muller et al., 2001, p.265). Portanto a atividade microbiana do rúmen dos bovinos pode ser considerada eficaz em degradar grande parte desta micotoxina, consequentemente se a quantidade não for muito alta na dieta, a fermentação ruminal protege os animais contra os seus efeitos tóxicos (Battacone et al., 2010, p. 1809).

\section{TRICOTECENOS}

Os Tricotecenos também são degradados no rúmen para compostos menos tóxicos. Tricotecenos como, toxina-T2, deoxivalenol (também descrito como vomitoxina) e diacetoxiscirpenol produzidos por espécies de Fusarium são conhecidos por serem convertidos em larga escala no rúmen para de-epoxi-tricotecenos (Fink-Gremmels, 2008a, p. 174) que são compostos menos tóxicos.

Foram testadas in vitro a capacidade de degradação deoxinivalenol de 29 estirpes de bactérias produtoras de ácido láctico e propiônico. Foi observada alta variabilidade na capacidade de degradação entre as bactérias. Para as produtoras de ácido lático as que apresentaram maior degradação do deoxivalenol foram Lactobacillus delbruekii ssp. Bulgaricus (55\%) e para as produtoras de ácido propiônico foram Propionibacterium (18\%). Também foi observado que algumas bactérias não degradam essa micotoxina (Lactobacillus helveticus, Pediococcus acidilactici, Pediococcus pentosaceus) (Niderkorn et al., 2006, p. 854). Assim a eficiência da degradação dos tricotecenos provavelmente tem influência do tipo da dieta fornecida aos bovinos, pois esta interfere na população microbiana e consequentemente na degradação desta micotoxina.

Os tricotecenos podem ser tóxicos para alguns tipos de bactérias. Em um trabalho de Dänicke et al. (2002, p. 245) foi verificado que o fornecimento de trigo contaminado com toxinas produzidas por Fusarium (10 mg deoxinivalenol e $0,76 \mathrm{mg}$ zearalenona por $\mathrm{kg}$ de $\mathrm{MS}$ ) causou redução na síntese de proteína microbiana em $24 \%$ e no fluxo de proteína total para duodeno em $25 \%$, o que demonstra que os tricotecenos podem ser tóxicos para alguns microrganismos podendo assim alterar o metabolismo ruminal.

\section{ZeARALENONA}

A zearalenona é convertida pelos microrganismos ruminais para $\alpha$-zearalenol (aproximadamente 90\%) e em menor quantidade para $\beta$-zearalenol (Kennedy et al., 1998, p. 393). O $\alpha$-zearalenol tem um potencial estrogênico maior que a molécula de zearalenona original, porém este primeiro apresenta uma menor taxa de absorção (Seeling et al., 2005, p.847), o que pode explicar a maior tolerância dos bovinos a esta micotoxina em relação aos monogástricos (Fink-Gremmels, $2008^{a}$, p. 174).

Suspeitando que a zearalenona pudesse alterar a fermentação ruminal Riccio et al. (2014, p. 309) conduziram um experimento in vitro para testar diferentes doses de zearalenona (0, 5 e $10 \mathrm{mg} / \mathrm{L})$ sobre algumas variáveis ruminais. Não foram observadas alterações no $\mathrm{pH}$ do líquido ruminal, ácidos graxos voláteis, nitrogênio amoniacal e síntese de proteína microbiana. Porém houve redução na digestibilidade da fibra em detergente neutro $(28,9 \%, 19,8 \%$ e $10,9 \%$ para 0,5 e $10 \mathrm{mg} / \mathrm{L}$, respectivamente) e da fibra em detergente ácido $(32,8 \%, 22,2 \%$ e $12,8 \%$ para 0,5 e $10 \mathrm{mg} / \mathrm{L}$, res- 
pectivamente). Esses resultados podem ser atribuídos a um possível efeito tóxico da zearalenona às bactérias celulolíticas do rúmen.

\section{PATULINA}

Dentre as micotoxinas a patulina provavelmente é a que exerce maior atividade antimicrobiana no rúmen, atuando sobre bactérias gram-positivas, gram-negativas e protozoários, afetando negativamente a fermentação ruminal (Escoula, 1992, p. 45). Em experimentos in vitro a patulina foi totalmente degradada no rúmen após 72 horas de incubação (Riccio et al., 2014, p. 309). Porém, apesar disso foi verificado que esta pode prejudicar a fermentação ruminal. Em outros experimentos in vitro a inclusão desta micotoxina reduziu a produção total de AGV (principalmente de acetato), proteína microbiana (Escoula, 1992, p. 45; Tapia et al., 2002, p. 239), degradação da matéria seca (Morgavi et al., 2003, p. 6906) e FDN e FDA (Riccio et al., 2014, p. 309). Assim esses resultados mostram que a patulina é altamente degradada no rúmen, porém devido a sua alta atividade antimicrobiana o metabolismo ruminal é negativamente alterado.

\section{FUMONISINAS}

As fumonisinas são pouco degradadas no rúmen. Em um estudo in vitro avaliando o efeito da fumonisina B1 no metabolismo ruminal, foi observada uma mínima degradação desta micotoxina (menos de 100 ppm do material da cultura) pelos microrganismos do líquido ruminal ao longo de um período de 72 horas e nenhum efeito sobre a produção microbiana (Gurung et al., 1999, p. 196). Em outro experimento in vitro a utilização de fumonisina B1 (1 $\mu \mathrm{g} / \mathrm{mL}$ de liquido ruminal) também por 72 horas de incubação não alterou a produção de ácidos graxos voláteis e houve redução de até $18 \%$ na concentração de fumonisina B1 adicionada. Estes resultados indicam que as fumonisinas são pouco metabolizadas no rúmen e sugerem que a alta tolerância dos bovinos a esta micotoxina não é causada pela biodegradação no rúmen (Caloni et al., 2000, p.379).

\section{AlCALOIDES DE ERGOT}

Os alcaloides de Ergot possuem maior importância para ruminantes por serem produzidos principalmente em forrageiras. O Ergot pertence a uma classe de alcaloides produzidos por fungos que não são inativados pelos microrganismos ruminais. Os fungos Neothyphodium spp. e Claviceps spp. produzem uma gama de alcaloides, sendo o principal deles o Ergot, e que altera o metabolismo ruminal (Rodrigues, 2014, p. 1159). Em um experimento com vacas canuladas no rúmen o fornecimento de Ergot $(41,1$ a 16,3 $\mu \mathrm{g} / \mathrm{kg}$ de peso corporal) causou alterações nas produções de isovalerato, propionato e nitrogênio amoniacal o que pode indicar uma mudança na população bacteriana. Além disso, a temperatura retal das vacas aumentou significativamente após o fornecimento de Ergot, o que leva a efeitos negativos em animais estressados por calor (Schumann et al., 2008, p. 57). Em um outro estudo, o fornecimento 169 e 433 ppb de alcaloides de ergot em relação ao controle (3 ppb) para ovelhas, apesar de não terem reduzido a digestibilidade da matéria seca, materia orgánica e proteína bruta, reduziram as digestibilidades da fibra em detergente neutro e ácido, revelando que estes compostos podem atuar sobre bacterias fibrolíticas (Coufal-Majewski et al., 2017, pp. 103).

Dessa forma não podemos generalizar que a fermentação ruminal é eficiente em degradar as micotoxinas consumidas por bovinos. Como foi supracitado, a eficiência na degradação depende além do tipo e da quantidade de micotoxina ingerida, do tipo biológico do animal, da dieta e tipos de microrganismos que estão colonizando o rúmen. Algumas micotoxinas são altamente biodegradadas, outras não e algumas ainda são altamente toxicas para o microrganismos alterando negativamente o metabolismo ruminal.

\section{METABOLISMO PÓS-ABSORTIVO E SINAIS CLÍNICOS DE INTOXICAÇÃO}

\section{AfLATOXINAS}

Após sua absorção, as aflatoxinas são metabolizadas no fígado. Reações de hidroxilação levam a formação de aflatoxinas como a M1 e Q1, dentre outras, que por possuir um grupamento hidroxila na molécula, tornam-se bastante solúveis em água, possibilitando sua rápida excreção através da urina, bile (consequentemente pelas fezes) e leite (Oliveira e Germano, 1997, p. 418; Nidhina et al., 2017, pp. 26). Reações de epoxidação por outro lado, levam a formação de compostos ativados, como o 8,9-óxido de AFB1 e o AFB1-2,3 epóxido, compostos altamente eletrofílicos, capazes de reagir de forma covalente com macromoléculas de DNA, RNA e proteínas. Sua ligação modifica a estrutura e a atividade biológica do DNA e de proteínas, provocando alterações mutagênicas na célula, que por sua vez leva a ocorrência de lesões bioquímicas, com inativação de macromoléculas essenciais e morte celular, originando os efeitos básicos da intoxicação (Oliveira e Germano, 1997, p. 418).

Essas micotoxinas são potentes hepatotóxicos, apresentando também propriedades carcinogênicas, mutagênicas e imunossupressoras. Os sinais clínicos de aflatoxicose crônica incluem redução da produção de leite em bovinos leiteiros, baixos ganhos de peso em animais em crescimento, redução no consumo de matéria seca, danos ao fígado, com elevação das enzimas hepáticas e bilirrubina, dores abdominais e cólica, diarreia, tenesmo, podendo estar acompanhado por prolapso de reto e imunocompetência reduzida. Aflatoxicoses agudas são raras, porém podem provocar fotodermatite, edema submandibular, diarreia (às vezes sanguinolenta) e alterações nervosas. Embora existam relatos na literatura, as Aflatoxinas normalmente não levam a ocorrência de abortos e problemas reprodutivos (Awad et al., 2012, p. 1; Riet-Correa et al., 2013, p. 692).

\section{OCRATOXINA A}

Apesar da elevada capacidade de metabolização da ocratoxina A pelos microrganismos ruminais, sua conversão em Ocratoxina $\alpha$ é incompleta, tendo sido detectada a presença desta micotoxina na urina, sangue e no leite de bovinos e ovinos (Hohler et al., 1999, 
pflądXXi, p. 6690). A ocratoxina A não metabolizada pode ser absorvida no intestino delgado, se ligando fortemente as proteínas séricas, principalmente a Albumina, sendo assim carreada para os diferentes tecidos corporais, porém os rins são os principais tecidos alvos (Sorrenti et al., 2013, p. 1744).

Diversos mecanismos foram propostos para a ocratoxina $\mathrm{A}$, que podem variar entre os diferentes tecidos corporais. Esta micotoxina atua como inibidor da síntese proteica devido ao seu efeito sobre as enzimas fenilalanina t-RNA sintetase e fenilalanina hidroxilase por meio de mecanismos não específicos, podendo ter influência sobre a transcrição de diversas proteínas, com efeitos adversos no meio intracelular. A exposição à ocratoxina A leva também a redução na atividade de enzimas chave da gliconeogênese (p. ex. fosfoenolpiruvato carboxiquinase; PEPCK), devido a sua interferência na expressão da PEPCK em termos de mRNA, pela ligação a proteínas envolvidas na manutenção do potencial da membrana no interior da mitocôndria e interferindo no transporte de fosfato e de elétrons na fosforilação oxidativa (Meisner e Chan, 1974, p. 2799; Thekkumkara e Patel, 1989, p. 917). A oxidação desta micotoxina pode gerar produtos eletrofílicos chamados ocratoxina A-quinona, que podem se ligar de forma covalente ao DNA, levando a ocorrência de mutações e subsequentemente formação de tumores. Estudos indicaram também que a exposição a esta micotoxina leva a produção de radicais livres em excesso, aumentando o estresse oxidativo nas células, provocando danos aos lipídios, proteínas e ao DNA (Koszegi e Poór, 2016, p. 117).

A ocratoxina A apresenta uma ampla gama de efeitos neurotóxicos, hepatotóxicos, teratogênicos, carcinogênicos e imunotóxicos (Koszegi e Poór, 2016, p. 112). Para bovinos adultos o fornecimento de uma única de dose de ocratoxina A (13 mg/kg de peso corporal) ocasionou sinais clínicos como desenvolvimento de anorexia, redução na produção de leite, diarreia e falta de coordenação (Meerdink et al., 2004, p. 235).

\section{TRICOTECENOS}

Doses elevadas de tricotecenos induzem a apoptose de leucócitos, células T, células B e imunoglobulina A. Esses compostos se ligam a receptores nos ribossomos dessas células, induzindo à fosforilação e ativação de proteínas quinase, que interferem de forma negativa em diversos processos fisiológicos, reduzindo o crescimento e diferenciação celular, podendo acarretar em sua apoptose, e supressão da resposta imune (Pestka et al., 2004, p. 61). Esta micotoxina se apresenta como um potente imunossupressor, atuando na inibição da síntese proteica e interferindo na síntese de DNA e RNA, com efeitos principalmente sobre células de elevada atividade metabólica e multiplicação, induzindo a ocorrência de dermatites e lesões ulcerativas no trato gastrointestinal. Sintomas de toxicidade incluem desconforto abdominal, salivação, diarreias, vômito e anorexia, com consequente redução na ingestão de alimentos, ganho de peso e eficiência alimentar (Pestka,

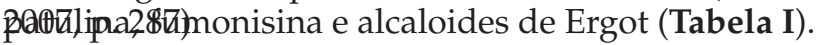

\section{ZEARALENONA}

A estrutura química da zearalenona, assim como a de seus metabólitos ( $\alpha$-zearalenol, $\beta$-zearalenol, $\alpha$-zearalenol e $\beta$-zearalenol) permitem a ligação desses compostos a receptores estrogênicos em mamíferos, levando a alterações em processos mediados por esteroides pela neutralização ou imitação de hormônios naturais (Krížová et al., 2012). Dessa forma a zearaleona pode induzir a alterações no trato reprodutivo dos animais, peso das glândulas adrenais, tireoide e pituitária, e níveis séricos de progesterona e estradiol, resultando em maior dificuldade para a concepção, ovulação, implantação e desenvolvimento do feto e de animais recém-nascidos. Estes normalmente não apresentam efeitos teratogênicos (Kuiper-Goodman et al. 1987, p. 279; Kennedy et al., 1998, p. 393).

Winkler et al. (2014, p. 196) avaliaram o fornecimento de diferentes níveis de zearalenona $(0,2$ a $0,66 \mathrm{mg} /$ $\mathrm{kg}$ de MS) associada a diferentes níveis de deoxivalenol para vacas e não observaram relação com o desempenho (produção de leite, mudança de peso e consumo de matéria seca).

\section{PATULINA}

Em uma revisão com diversas espécies de animais foi observado uma ampla gama de alterações celulares causadas pela patulina, como danos na membrana plasmática, inibição da síntese proteica, inibição dos transportadores de aminoácidos dependentes de sódio, interrupção da transcrição e tradução, inibição da síntese de DNA, etc. Os principais sinais de intoxicação aguda por patulina incluem agitações, convulsões, dispneia, congestão pulmonar, edema, ulceração, hiperemia, distensão trato gastrointestinal, hemorragia intestinal, degeneração das células epiteliais, inflamação intestinal e outros danos gastrointestinais e renais. Os sintomas de intoxicação crônica incluem efeitos neurotóxicos, imunotóxicos, imunossupressor, genotóxicos, teratogênicos e efeitos cancerígenos (Moake et al., 2005, p. 9). Porém em ruminantes a patulina é altamente degradada no rúmen, como foi descrito anteriormente, e assim além das alterações na fermentação ruminal e possíveis danos à parede do rúmen os riscos de intoxicação por essa micotoxina são baixos.

\section{FUMONISINAS}

As fumonisinas apresentam uma cadeia longa de hidrocarbonetos, similar a esfingosina e esfinganina, sendo este, fator fundamental em seu mecanismo de toxicidade, por provocar a interrupção do metabolismo dos esfingolipídios (Hussein e Brasel, 2001, p. 105). Sua ação ocorre independentemente de ativações metabólicas. Este metabólito apresenta um grupamento amino no carbono 2 de sua estrutura que inibe de forma competitiva a enzima ceramida sintetase, uma enzima chave na biossíntese dos esfingolipídios, acarretando no acúmulo do precursor esfinganina e redução na concentração de esfingosina, apresentando como resultado a interrupção da biossíntese de novo de ceramida e o metabolismo de esfingolipídios. A interrupção na síntese de esfingolipídios interfere no crescimento e diferenciação celular, resultando em toxicidade e car- 
cinogenicidade (Glenn, 2007, p. 220; Voss et al., 2007, p. 309).

Intoxicação por fumonisinas em bovinos são descritas por causar injuria renal e hepática, com elevação nos níveis séricos de enzimas específicas desses órgãos, assim como níveis elevados de colesterol e bilirrubina (Fink-Gremmels, 2008a, p. 175). Porém ruminantes são amplamente tolerantes a fumonisina, ocorrendo mínima absorção desta. Mais de $80 \%$ do total ingerido não é metabolizado pelos microrganismos ruminais e é excretada nas fezes e apenas traços desse metabólito são encontrados na urina de bovinos (Smith e Thakur, 1996, p. 39).

Osweiler et al. (1993, p. 462) forneceram níveis crescentes de fumonisina a bezerros e observaram que apenas a dose mais elevada (148 ppm) levou a incrementos nas concentrações das enzimas hepáticas e lesões leves nesse órgão, não sendo observados efeitos negativos sobre o consumo de matéria seca e ganho de peso.

\section{AlCALOIDES DE ERGOT}

Sua atividade biológica e efeitos fisiológicos são dependentes de sua estrutura e propriedades, que se assemelham a das aminas biogênicas, como norepinefrina, epinefrina, dopamina e serotonina. Esses compostos se ligam aos receptores dopaminérgicos, serotoninérgicos e $\alpha$-adrenérgicos que atuam na regulação das funções cardiovasculares, endócrina, motilidade do trato gastrointestinal, contração da musculatura lisa, regulação da temperatura corporal e apetite, sendo os sintomas de intoxicação resultantes das alterações nessas vias regulatórias (Strickland et al., 2011, p. 1604). As intoxicações por alcaloides de Ergot em bovinos estão associadas principalmente a dois distúrbios metabólicos denominados "Summer Slump" e "Fescue Foot" (Hussein e Brasel, 2001, p. 107).

O "Summer Slump" é conhecido como o efeito mais significativo da intoxicação por Alcaloides de Ergot em bovinos, levando a baixas taxas de crescimento, pelagem grosseira devido à manutenção da pelagem de inverno, elevação da temperatura corporal, maior frequência respiratória e salivação excessiva, sendo os sintomas agravados em temperaturas ambientais elevadas. Esses sintomas são associados à dificuldade na dissipação do calor corporal nesses animais, provocados pela vasoconstrição periférica, que leva a alterações comportamentais, redução no consumo e desempenho dos animais (Spiers et al., 2005, p. 1423).

O distúrbio "Fescue foot" também está associado à vasoconstrição periférica proporcionada pela intoxicação por alcaloides de Ergot, sendo seus sintomas mais comuns durante o inverno, levando a inchaço e claudicação, principalmente nas patas traseiras, resultante da associação da vasoconstrição natural que ocorre em função da termorregulação, induzida pelas toxinas, e como consequência a condições gangrenosas nas extremidades e necrose (Spiers et al., 2005, p. 1423).

Estudos com ovinos mostraram que o fornecimento de alcaloides de Ergot reflete em maior enchimento do trato digestivo. Essa resposta tem sido associada à redução da circulação sanguínea no trato gastrointestinal, redução da motilidade do retículo-rúmen e elevada contração tônica nesses órgãos. Essas respostas podem ser decorrentes da ação direta dessas toxinas sobre os neurônios mioentéricos e na musculatura lisa, reduzindo assim a motilidade, taxa de passagem, e a ingestão de alimentos (McLeay e Smith, 2006, p. 707).

Os Alcaloides de Ergot podem afetar o desempenho reprodutivo, por causar vasoconstrição local e decréscimo no fluxo sanguíneo para tecidos reprodutivos, redução do consumo de matéria seca e elevadas temperaturas corporais, porém ainda não tem sido reportado de forma consistente qual é o real impacto dessas toxinas sobre o desempenho reprodutivo em bovinos de corte (Porter e Thompson Jr., 1992, p. 1594; Strickland et al., 2011, p. 1614).

\section{MÉTODOS DE PREVENÇÃO, CONTROLE E DETOXIFI- CAÇÃO}

Os métodos de prevenção mais eficazes são realizados anteriormente ao desenvolvimento dos fungos no material vegetal. Várias práticas agrícolas podem ser utilizadas para diminuir a contaminação, porém mesmo as melhores estratégias de gestão nem sempre são suficientes para erradicar totalmente a produção de fungos e de suas micotoxinas.

No período pré-colheita dos grãos algumas estratégias podem ser utilizadas para diminuir a contaminação por micotoxinas como: rotação de culturas, quantidade de fertilizantes utilizados (altera a taxa de decomposição dos resíduos, taxa de crescimento da planta, estrutura e atividade microbiana do solo), escolha de cultivares menos susceptíveis ao desenvolvimento de Fusarium spp, data de plantio (contaminação é maior quando a fase de floração das culturas ocorre no momento da liberação dos esporos), uso de fungicidas (esses devem ser altamente letais contra Fusarium spp, caso contrário podem estimular a produção de micotoxinas), controle biológico (antagonistas microbianos, como, por exemplo, Bacillus subtilis quando pulverizado na fase de florescimento pode inibir o crescimento de fungos durante a fase de crescimento endofítico), controle de insetos (comprometem a proteção externa dos grãos e os tecidos da planta, permitindo que as hifas de fungos penetrem e tenham acesso aos nutrientes, além disso os insetos carregam os esporos dos fungos), controle de ervas daninhas dentre outros (Jouany, 2007, p. 343).

A contaminação por fungos do gênero Fusarium pode ocorrer já nos primeiros estágios de desenvolvimento da espiga do milho e a produção de fumonisinas aumentam ao longo do desenvolvimento e maturação fisiológica dos grãos. Assim o momento da colheita dos grãos pode ter consequência no nível final de contaminação, e colheitas mais tardias normalmente apresentam maior contaminação. Além disso, outros fatores como regulagem da colheitadeira, umidade dos grãos antes e durante o armazenamento, temperatura durante a armazenagem também influenciam na concentração final de micotoxinas nos cereais (Jouany, 2007, p. 345; Gallo et al., 2015, p. 3088).

A silagem de milho pode conter micotoxinas como aflatoxinas, tricotecenos, ocratoxina A, fumonisinas, 
zearalenona e diversos outros metabólitos secundários de origem fúngica (Ogunade et al., 2018, p. 4034). Alguns cuidados podem reduzir a produção de micotoxinas nas silagens. $\mathrm{O}$ teor de umidade da planta inteira no momento da ensilagem deve estar entre 65 a $70 \%$ para silos tipo trincheira ou cobertura, o silo deve ser preenchido rapidamente, compactado e selado completamente. $\mathrm{O}$ tamanho do silo deve ser ajustado para que a remoção diária da parte frontal seja de no mínimo $12,9 \mathrm{~cm}$. A utilização de aditivos biológicos ou químicos que melhorem a fermentação e diminuam o pH rapidamente podem ser utilizados para reduzir o crescimento de fungos. Também podem ser utilizados fungicidas na parte externa do silo anterior a vedação ou na parte frontal do silo após a retirada diária (Jouany, 2007 , p. 350). As práticas de produção e utilização de silagem devem evitar ao máximo a permanência ou entrada de oxigênio no silo, pois este favorece o desenvolvimento de microrganismos aeróbicos que incluem os fungos (Gallo et al., 2015, p. 3088).

Como é muito difícil prevenir totalmente a produção de micotoxinas tanto no período pré-colheita como no armazenamento, algumas ferramentas/alternativas foram desenvolvidas para proteger os animais da ingestão de alimentos contaminados. Pode ser feita a classificação ou separação dos grãos inteiros dos quebrados, estes últimos normalmente apresentam maior contaminação, lavagem, tratamento térmico como por exemplo floculação, irradiação, esta pode ser utilizada para descontaminação de esporos de fungos mas não para a redução de micotoxinas já produzidas, tratamento químico, como por exemplo hidróxido de amônio para reduzir aflatoxina e os adsorventes de micotoxinas, como argilas, carvão ativado e produtos da parede celular de leveduras (Jouany, 2007, p. 350; Vila-Donat et al., 2018, p. 247). Estes agentes sequestrantes de micotoxinas são compostos capazes de se ligar as micotoxinas sem dissociar, permitindo que estas passem pelo trato gastrointestinal sem serem absorvidas e são eliminadas nas fezes (Gallo et al., 2015, p. 3090; Vila-Donat et al., 2018, p. 249). A eficiência desses compostos ainda são controversas e assim a maior mobilização de recursos deve ser direcionada para prevenir o desenvolvimento de fungos e de seus compostos secundários.

A produção de grãos de cereais ou silagem nem sempre é possível de ser feita com todos os cuidados supracitados. Assim quando houver suspeita da presença de altas concentrações de micotoxinas, deve-se coletar uma amostra representativa do lote, e enviar para análise em laboratório. Se for identificada altas concentrações de alguma micotoxina específica podem ser tomadas atitudes como: fornecer para espécies ou categorias mais resistentes a aquela micotoxina específica; reduzir a quantidade fornecida do lote contaminado aos animais, proporcionando assim que consumo diário da micotoxina identificada esteja dentro de uma faixa segura; ou até mesmo decidir sobre a utilização de adsorventes ou aplicação de algum método de degradação de micotoxinas como foi descrito anteriormente.

\section{CONCLUSÕES}

O rúmen dos bovinos causa alterações na estrutura da maioria das micotoxinas consumidas, isto muitas vezes reduz a toxidez deste composto para o animal. Porém as micotoxinas na sua grande maioria apresen- tam efeito antimicrobiano e isto pode causar efeitos negativos na fermentação ruminal e consequentemente prejuízos no desempenho e lucratividade da atividade.

Regiões tropicais normalmente possuem condições edafoclimáticas ideais para o desenvolvimento de fungos e consequentemente de seus metabolitos secundários. As altas temperaturas no período produtivo dos cereais associado à alta umidade são condições excelentes para produção de micotoxinas tanto no período pré-colheita como no armazenamento. Assim é de estrema importância que os produtores rurais adotem cada vez mais medidas que minimizem a produção destes compostos.

\section{BIBLIOGRAFIA}

Auerbach, H, Oldenburg, E, Weissbach, F 1998, 'Incidence of Penicillium roqueforti and roquefortine $C$ in silages', Journal of the Science of Food and Agriculture, vol. 76, pp. 565-572.

Awad, W.A, Ghareeb, K, Böhm, J 2012, 'Occurrence, Health Risks and Methods of Analysis for Aflatoxins and Ochratoxin A', Journal of Veterinary Science, vol. 2, pp. 1-10.

Balzer, A, Tardieu, D, Bailly, J.D, Guerre, P 2004, 'The trichothecenes: the nature of toxins, natural occurrence in foods and feeds and ways of combating their occurrence', Revue de Médecine Vétérinaire, vol.155, pp. 299-314.

Battacone, G, Nudda, A, Pulina, G 2010, 'Effects of Ochratoxin A on Livestock Production', Toxins, vol. 2, pp. 1796-1824.

Binder, E.M, 2007, Managing the risk of mycotoxins in modern feed production', Animal Feed Science and Technology, vol. 133, pp. 149-166.

Binder, E.M, Tan, L.M, Chin, L.J, Handl, J, Richard, J 2007, 'Worldwide occurrence of mycotoxins in commodities, feeds and feed ingredients', Animal Feed Science and Technology, vol.137, pp. 265-282.

Blank, R, Rolfs, J.P, Sudekum, K.H, Frohlich, A.A, Marquardt, R.R, Wolffram, S 2003. 'Effects of chronic ingestion of ochratoxin A on blood levels and excretion of the mycotoxin in sheep', Journal of Agricultural and Food Chemistry, vol. 51, pp. 6899-6905.

Blount, W.P 1960, 'Disease of turkey poults', Veterinary Record, vol. 72, pp.786

Blount, W.P 1961, 'Turkey "X" disease', Turkeys, vol. 9, pp. 52-55. Boudra, H, Barnouin, J, Dragacci, S, Morgavi, D.P 2007, 'Aflatoxin $M_{1}$ and ochratoxin $A$ in raw bulk milk from French dairy herds'. Journal of Dairy Science, vol. 90, pp. 3197-3201

Boudra, H, Saivin, S, Buffier E.C, Morgavi, D.P 2013, 'Short communication: Toxicokinetics of ochratoxin A in dairy ewes and carryover to milk following a single or long-term ingestion of contaminated feed', Journal of Dairy Science, vol. 96, pp. 6690-6696.

Cook, W.O, Richard, J.L, Osweiler, G.D, Trampel, D.W 1986, 'Clinical and pathologic changes in acute bovine aflatoxicosis: rumen motility and tissue and fluid concentrations of aflatoxins B 1 and $\mathrm{Ml}^{\prime}$, American Journal of Veterinary Research, vol. 47, pp. 1817-1825.

Coufal-Majewski, S, Stanford, K, McAllister, T, Wang, Y, Blakley, B, McKinnon, J, Chaves, A.V 2017, 'Effects of pelleting diets containing cereal ergot alkaloids on nutrient digestibility, growth performance and carcass traits of lambs', Animal Feed Science and Technology, vol. 230, pp.103-113.

Caloni, F, Spotti, M, Auerbach H, Op Den Camp, H, Gremmels, J.F, Pompa, $G$ 2000, 'In vitro metabolism of fumonisin B1 by ruminal microflora', Veterinary Research Communications, vol. 24, pp. 379-387.

Castelo, M.M, Bullerman, L.B 2001, 'Reduction of fumonisin by the corn flake process', In: Proceedings of the American Association of Cereal Chemists Annual Meeting, Charlotte, North Carolina, 14-18 Outubro (Abst. no. 362). 
Dänicke, S, Gadeken, D, Ueberschar, K.H, Meyer, U, Scholz, H 2002, 'Effects of Fusarium toxin contaminated wheat and of a detoxifying agent on performance of growing bulls, on nutrient digestibility in wethers and on the carry over of zearalenone', Archiv fur Tierernahrung, vol. 56, pp. 245-261

Escoula, L 1992, 'Patulin production by Penicillium granulatum and inhibition of ruminal flora', Journal of Environmental Pathology, Toxicology and Oncology, vol. 11, pp. 45-48.

Fink-Gremmels, J 2008a, 'Mycotoxins in cattle feeds and carry-over to dairy milk: a review', Food Additives and Contaminants, vol. 25, pp. 172-180.

Fink-Gremmels, J 2008b. 'The role of mycotoxins in the health and performance of dairy cows', Veterinary Journal, vol. 176, pp. 84-92.

Frank, H.K, Grunewald, T 1970, 'Radiation resistance of aflatoxins', Irradiat. Aliments, vol. 11, pp. 15-20.

Fruhmann, $P$, Mikula, $H$, Wiesenberger, $G$, Varga, E, Lumpi, D, Stoger, B, Haubl, G, Lemmens, M, Berthiller, F, Krska, R, Adam, G Hametner, C, Frohlich, J 2014, 'Isolation and Structure Elucidation of Pentahydroxyscirpene, a Trichothecene Fusarium Mycotoxin', Journal of Natural Products, vol. 77, pp. 188-192.

Gallo, A, Giuberti, G, Frisvad, J.C, Bertuzzi, T, Nielsen, K.F 2015, 'Review on mycotoxin issues in ruminats: Ocurrence in forages, effects of mycotoxin ingestion on health status and animal performance and practical strategies to counteract their negative effects', Toxins, vol. 7, pp. 3057-3111.

Glenn, A.E 2007, 'Mycotoxigenic Fusarium species in animal feed', Animal Feed Science and Technology, vol. 137, pp. 213-240.

Gurung, N.K, Rankins Jr., D.L, Shelby, R.A 1999, 'In vitro ruminal disappearance of fumonisin $\mathrm{B} 1$ and its effect on in vitro dry matter disappearance', Veterinary and human toxicology, vol. 41, pp. 196-199

Hohler, D, Sudekum, K.H, Wolffram, S, Frohlich, A.A, Marquardt, R.R 1999, 'Metabolism and excretion of ochratoxin A fed to sheep', Journal of Animal Science, vol. 77, pp. 1217-1223.

Hult, K, Teiling, A, Gatenbeck, S 1976, 'Degradation of ochratoxin A by a ruminant', Applied and Environmental Microbiology, vol. 32, pp. 443-444

Hussein, H.S, Brasel, J.M 2001, 'Toxicity, metabolism, and impact of mycotoxins on humans and animals', Toxicology, vol. 167, pp. 101-134

Jiang, Y.H, Yang, H.J, Lund, P 2012, 'Effect of aflatoxin B1 on in vitro ruminal fermentation of rations high in alfalfa hay or ryegrass hay', Animal Feed Science and Technology, vol. 175, pp. 85- 89.

Jouany, J.P 2007, 'Methods for preventing, decontaminating and minimizing the toxicity of mycotoxins in feeds', Animal Feed Science and Technology, vol. 37, pp. 342-362.

Kennedy, D.G, Hewitt, S.A, Mcevoy, J.D, Currie, J.W, Cannavan, A, Blanchflower, W.J, Elliot, C.T 1998, 'Zeranol is formed from Fusarium spp. Toxins in cattle in vivo', Food Additives and Contaminants, vol. 15, pp. 393-400.

Koszegi, T, Poór, M 2016, 'Ochratoxin A: Molecular Interactions, Mechanisms of Toxicity and Prevention at the Molecular Level', Toxins, vol. 8, no. 4, pp. 111-135.

Kuiper-Goodman, T, Scott, P.M, Watanabe, H 1987, 'Risk assessment of the mycotoxin zearalenone'. Regulatory Toxicology and Pharmacology, vol. 7, pp. 253-306.

Krízová, L, Pavlok, S, Vesely, A 2012, 'The fate of feedborne mycotoxin zearalenone (ZEA) in dairy cows - a review', Vyzkum v chovu skotu/ Cattle Research, vol. 3

Mcleay, L.M, Smith, B.L 2006, 'Effects of ergotamine and ergovaline on the electromyographic activity of smooth muscle of the reticulum and rumen of sheep', American Journal of Veterinary research, vol. 67, pp. 707-714.

Meerdink GL 2004, 'Citrinin and ochratoxin', In: Plumlee KH, editor. Clinical veterinary toxicology. St Louis (MO): Mosby; p. 235-9.

Meisner, H, Chan, S 1974, 'Ochratoxin A, an Inhibitor of Mitochondrial Transport Systems', Biochemestry, vol. 13, no. 14, pp. 2795-2800.
Moake, M.M, Padilla-Zakour, O.I, Worobo, R.W 2005, 'Comprehensive Review of Patulin Control Methods in Foods', Comprehensive Reviews in Food Science and Food Safety, vol. 1. pp. 8-21.

Mobashar, M, Hummel, J, Blank, R, Sudekum, K.H 2010, 'Ochratoxin $A$ in ruminants - a review on its degradation by gut microbes and effects on animals', Toxins, vol. 2, pp. 809-839.

Morgavi, D, Boudra, H, Jouany, J, Graviou, D 2003, 'Prevention of patulin toxicity on rumen microbial fermentation by SH-containing reducing agents'. Journal of Agriculture and Food Chemistry, vol. 51, pp. 6906-6910.

Moss, M.O, 1991, 'The environmental factors controlling mycotoxin formation'. In: Smith, J.E., Anderson, R.A. (Eds.), Mycotoxins and Animal Foods. CRC Press, Boca Raton, FL, pp. 37-56.

Mostrom, M.S, Jacobsen, B.J 2011, 'Ruminant Mycotoxicosis', Veterinary Clinics of North America: Food Animal Pratice, vol. 27, pp. 315-344.

Muller, H.M, Muller, K, Steingass, H 2001, 'Effect of feeding regime on the metabolism of ochratoxin A during the in vitro incubation in buffered rumen fluid from cows'. Archiv für Tierernaehrung, vol. 54, pp. 265-279.

Niderkorn, V, Boudra, H, Morgavi, D.P 2006, 'Binding of Fusarium mycotoxins by fermentative bacteria in vitro', Journal of Applied Microbiology, vol. 101, pp. 849-856.

Nidhina, N, Bhavya, M.L, Bhaskar, N, Muthukumar, S.P, Murthy, P.S 2017, 'Aflatoxin production by Aspergillus flavus in rumen liquor and its implications', Food Control, vol. 71, pp. 26-31.

Norred, W.P, Voss, K.A, Bacon, C.W, Riley, R.T 1991, 'Effectiveness of ammonia treatment in detoxification of fumonisin-contaminated corn', Food and Chemical Toxicology, vol. 29, pp. 815-819.

Ogunade, I.M, Jiang, Y, Kim, D.H, Pech Cervantes, A.A, Arriola, K.G, Vyas, D, Weinberg, Z.G, Jeong, K.C, Adesogan, A.T, 2017, 'Fate of Escherichia coli $\mathrm{O} 157: \mathrm{H7}$ and bacterial di-versity in corn silage contaminated with the pathogen and treated with chemical or microbial additives', Journal of Dairy Science, vol. 100, pp. 1780-1794.

Oliveira, C.A.F, Germano, P.M.L 1997, 'Aflatoxinas: conceitos sobre mecanismos de toxicidade e seu envolvimento na etiologia do câncer hepático celular', Revista de Saúde Pública, vol. 31, no. 4, pp. 417-424.

Osweiler, G.D, Kehrli, M.E, Stabel, J.R, Thurston, J.R, Ross, P.F, Wilson, T.M 1993, 'Effects of fumonisin contaminated corn screenings on growth and health of feeder calves', Journal of Animal Science, vol. 71, pp. 459-466.

Pestka, J.J 2007, 'Deoxynivalenol: Toxicity, mechanisms and animal health risks', Animal Feed Science and Technology, vol. 137, pp. 283-298.

Pestka, J.J, Zhou, H, Moon, Y, Chung, Y.J 2004, 'Cellular and molecular mechanisms for immune modulation by deoxynivalenol and other trichothecenes: unraveling a paradox', Toxicology Letters, vol. 153, pp.61-73.

Placinta, C.M, D'Mello, J.P.F, Macdonald, A.M.C 1999, 'A review of worldwide contamination of cereal grains and animal feed with Fusarium mycotoxins', Animal Feed Science and Technology, vol. 78, pp. 21-37.

Porter, J.K, Thompson Jr., F.N 1992, 'Effects of fescue toxicosis on reproduction in livestock'. Journal of Animal Science, vol. 70, pp. 1594-1603.

Reisinger, N, Schürer-Waldheim, S, Mayer, E, Debevere, S, Antonissen, G, Sulyok, M, Nagl, V 2019, 'Mycotoxin Occurrence in Maize Silage-A Neglected Risk for Bovine Gut Health? ', Toxins, vol. 11 , pp. 1 - 17.

Riccio, M.B, Tapia, M.O, Martínez, G, Aranguren, S.M, Dieguez, S.N, Soraci, A.L, Rodríguez, E 2014, 'Effect of the combination of crude extracts of Penicillium griseo fulvum and Fusarium graminearum containing patulin and zearalenone on rumen microbial fermentation and on their metabolism in continuous culture fermenters', Archives of Animal Nutrition, vol. 68, pp. 309-319. 
Richard, J.L 2012, 'Mycotoxins - an overview', In 'Guide to mycotoxins featuring mycotoxin risk management in animal production'. (Ed. EM Binder) pp. 1-47. (Anytime Publishing Services: Leicestershire, UK).

Riet-Correa, F, Rivero, R, Odriozola, E, Adrien, M.L, Medeiros, R.M.T, Schild, A.L 2013, 'Mycotoxicoses of ruminants and horses', Journal of Veterinary Diagnostic Investigation, vol. 25, no. 6, pp. 692-708.

Rodrigues, I.A 2014, 'Review on the effects of mycotoxins in dairy ruminants', Animal Production Science, vol. 54, pp.1155-1 165.

Sargeant, K, Sheridan, A, O'Kelly, J, Carnahghan, R.B.A 1961, 'Toxicity associated with certain samples of groundnuts', Nature, vol. 192, pp. 1096-1097.

Schumann, B, Lebzien, P, Ueberschar, K.H, Spilke, J, Holtershinken, M, Danicke, S 2008, 'Effects of the level of feed intake and ergot contaminated concentrate on ruminal fermentation and on physiological parameters in cows', Mycotoxin Research, vol. 24, pp. 57-72.

Seeling, K, Danicke, S, Ueberschar, K.H, Lebzien, P, Flachowsky, G 2005, 'On the effects of Fusarium toxin-contaminated wheat and the feed intake level on the metabolism and carry over of zearalenone in dairy cows', Food Additives and Contaminants, vol. 22, pp. 847-855.

Smith, J.S, Thakur, RA 1996, 'Occurrence and fate of fumonisins in beef', Advances in Experimental Medicine and Biology, vol. 392, pp. 39-55.

Sorrenti, V, DI Giacomo, C, Acquaviva, R, Barbagallo, I, Bognanno, M, Galvano, F 2013, 'Toxicity of Ochratoxin A and its modulation by antioxidants: A review', Toxins, vol. 5, pp. 1742-1766.

Spiers, D. E.;.Eichen, P. A.; Rottinghaus, G. E. 2005, 'A model of fescue toxicosis: Responses of rats to intake of endophyte-infected tall fescue', Journal of Animal Science, vol. 83, pp. 1423-1434.

Sreemannarayana, O, Frohlich, A.A, Vitti, T.G, Marquardt, R.R, Abramson, D 1988, 'Studies of tolerance and disposition of ochratoxin $A$ in young calves', Journal of Animal Science, vol. 66, pp. 1703-1711.

Stevens, A.J, Saunders, C.N, Spence, J.B, Newham, A.C 1960, 'Investigations into "diseases" of turkey pults', Veterinary Record, vol. 72, pp. 627-628.

Strickland, J.R, Looper, M.L, Matthews, J.C, Rosenkrans JR, C.F, Flythe, M.D, Brown, K.R 2011 , 'Board-Invited Review: St. Anthony's Fire in livestock: Causes, mechanisms, and potential solutions'. Journal of Animal Science, vol. 89, pp. 1603-1626.

Tapia, M, Stern, M, Koski, T, Bach, A, Murphy, M 2002, 'Effects of patulin on rumen microbial fermentation in continuous culture fermenters', Animal Feed Science and Technology, vol. 97, pp. 239-246.

Thekkumkara, T.J, Patel, M.S 1989, 'Ochratoxin A decreases the activity of phosphoenolpyruvate carboxykinase and its mRNA content in primary cultures of rat kidney proximal convoluted tubule cells', Biochemical and Biophysical Research Comunications, vol. 162, no. 3, pp. 916-920.

Upadhaya, S.D, Sung, H.G, Lee, C.H, Lee, S.Y, Kim, S.W, Cho, K.J, $\mathrm{Ha}$, J.K 2009, 'Comparative study on the aflatoxin B1 degradation ability of rumen fluid from Holstein steers and Korean native goats', Journal of Veterinary Science, vol. 10, pp. 29-34.

Vila-Donat, P, Marin, S, Sanchis, V, Ramos, A.J 2018. `A review of the mycotoxin adsorbing agents, with an emphasis on their multi-binding capacity, for animal feed decontamination', Food Chem. Toxicol, vol. 114, pp. 246-259.

Voss, K.A, Smith, G.W, Haschek, W.M 2007, 'Fumonisins: Toxicokinetics, mechanism of action and toxicity', Animal Feed Science and Technology, vol.137, pp. 299-325.

Wilson, S.C, Brasel, T.L, Carriker, C.G, Fortenberry, G.D, Fogle, M.R, Martin, J.M, WU, C, Andriychuk, L.A, Karunasena, E, Straus, D.C 2004, 'An investigation into techniques for cleaning mould-contaminated home contents', Journal of Occupational and Environmental Hygiene, vol. 1, pp. 442-447.

Winkler, J, Kersten, S, Meyer, U, Engelhardt, U, Dänicke, S 2014, 'Residues of zearalenone (ZEN), deoxynivalenol (DON) and their metabolites in plasma of dairy cows fed Fusarium contaminated maize and their relationships to performance parameters'. Food and Chemical Toxicology, vol. 65, pp. 196-204. 